\title{
Research on the control effect of Beauveria bassian in common pests in
}

\section{frigid region}

\author{
Xue Chun-Mei ${ }^{1, a}$, Ren Guo-Li ${ }^{2, b}$, Sun Yan-Bin ${ }^{2, c}$, Zhang Yue-Hua ${ }^{2, d *}$ \\ ${ }^{1}$ College of Life Science, Jiamusi University Jiamusi, 154007, PR China; \\ ${ }^{2}$ College of Science, Jiamusi University Jiamusi, 154007, PR China \\ ajmsxcm@126.com, ${ }^{\mathrm{b}}$ chmqy215@163.com, ‘63145964@qq.com, \\ ${ }^{d *}$ Corresponding Author: zhangyaohua_2008@163.com
}

\begin{abstract}
Key words: Beauveria bassian; fungal suspension; LD $_{50}$; Fatality rate; Biopesticide
Abstract: Study on the control effect of $B$. bassiana on 4 kinds of pests of conservatory pests in frigid region. The content of $B$. bassiana was measured by plate counting method. B. bassiana was prepared into 5 concentrations of fungal suspension, named as $G_{1}$ to $G_{5}$, and $L_{50}$ lethal dose for experimental measurement standard, selecting median lethal concentration. The results showed that B. bassiana had significant effect on the death of 4 kinds of pests of conservatory, and $\mathrm{G}_{1}$ lethal rate was as high as $62 \%$; the lethality of $\mathrm{G}_{3}$ was $50 \% ; \mathrm{G}_{5}$ had the lowest mortality rate that was $34 \%$. Therefore, in the control of pests of conservatory in frigid region, B. bassiana, which was between $\mathrm{G}_{1}$ and $\mathrm{G}_{5}$, can be formulated as a biological pesticide for the control of conservatory damage by pests, but taking the control effect and the dosage of biological pesticides and many other factors into account, the experiment proved it was more suitable that concentration of B. bassiana strain $\mathrm{G}_{3}$ suspension.
\end{abstract}

\section{Introduction}

Due to the increasing of conservatory planting area in the northeast of the cold region, and the phenomenon of crop continuous cropping is prevalent, the outbreak of crop pests and diseases is increasing ${ }^{[1]}$. For a long time, the prevention and control of pests and diseases over-reliance on chemical pesticides, resulting in some pest and disease resistance was significantly increased, resulting in a vicious circle ${ }^{[2]}$. At the same time high chemical pesticide residues to cause harm to human body health, cause serious pollution to the ecological environment, destroy the ecological balance, this a series of problems spurred the search for a friendly to humans and the environment and has good control effect of plant diseases and insect pests prevention and control measures. As an emerging force in crop pest integrated control system, biological pesticides have become more and more prominent in the control of crop diseases and pests ${ }^{[3]}$. They have the characteristics of low toxicity, high efficiency and low residue, and are not easy to produce pest resistance ${ }^{[4]}$. B. bassiana is a more concerned class, its host a wide range of strong pathogenic, with a wide range of biological control ${ }^{[5]}$. In this paper, the $B$. bassiana suspension was prepared as a bio-pesticide by the culture of $B$. bassiana, and applied to four kinds of common pest parasites in cold conservatory. $\mathrm{LD}_{50}$ was used as the experimental standard to obtain the optimal lethal concentration, Provide the experimental basis for B. bassiana as a biological pesticide to control pests and diseases.

\section{Materials and Methods}

Powder

B. bassiana strains, Jiangxi Talent Ecology Co., Ltd.

Test insects

Trialeurodes vaporariorum, Brevicoryne brassicae, Pieris rapae and Plutella xylostella 4 
common conservatory pests were collected at the experimental planting base of vegetables in Jianan Experimental-Farm of Jiamusi University.

Preparation of PDA medium and PBS

PDA medium: $200.0 \mathrm{~g} / \mathrm{L}$ of potato (peeled), $20.0 \mathrm{~g} / \mathrm{L}$ of glucose, $20.0 \mathrm{~g} / \mathrm{L}$ of agar and $1.0 \mathrm{~L}$ of distilled water were placed in a $0.1 \mathrm{MPa}$ autoclave, sterilized for $20 \mathrm{~min}$ and stored at room temperature; PBS buffer: $\mathrm{NaCl} 8.00 \mathrm{~g}, \mathrm{KCl} 0.20 \mathrm{~g}, \mathrm{Na}_{2} \mathrm{HPO}_{4} 1.44 \mathrm{~g}$ dissolved in $800 \mathrm{~mL}$ distilled water, and adjust the $\mathrm{pH}$ of the solution to 7.4 with $\mathrm{HCl}$, constant volume to $1.0 \mathrm{~L}$, placed in $0.1 \mathrm{MPa}$ autoclave, sterilized 20min, stored at room temperature.

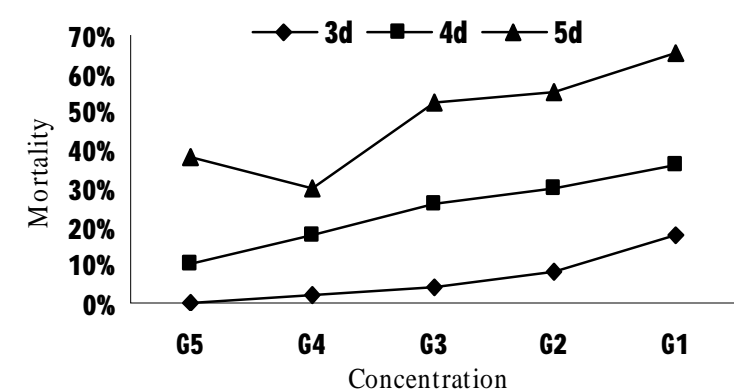

Figure 1. Lethality of Beauveria bassiana suspension to different concentrations of B. tabaci larvae

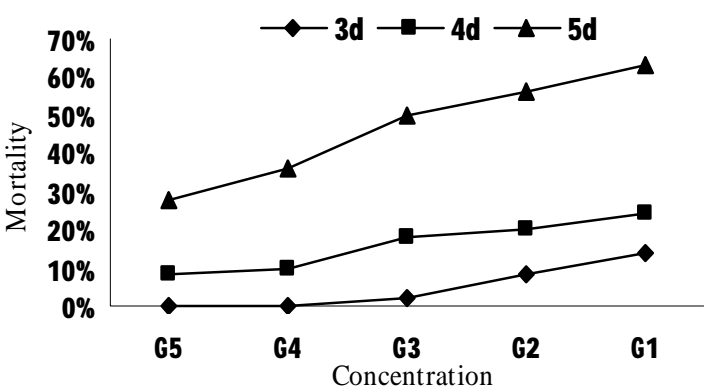

Figure 2. Different concentrations of Beauveria bassiana suspension to Gan Blue-eyed lethality

B. bassiana strain culture

Analytical balance accurately weighed $0.1000 \mathrm{~g}$ B. bassiana powder was dissolved in $10.0 \mathrm{~mL}$ distilled water and shock $3 \mathrm{~min}$, to obtain a concentration of $1 \times 10^{-2} \mathrm{~g} / \mathrm{mL}$ bacterial suspension, take $1.0 \mathrm{~mL} 1 \times 10^{-2} \mathrm{~g} / \mathrm{mL}$ of the bacterial suspension was dissolved in $9.0 \mathrm{~mL}$ of distilled water and shaken for $3 \mathrm{~min}$ to obtain a bacterial suspension having a concentration of $1 \times 10^{-3} \mathrm{~g} / \mathrm{mL}$. This step was repeated and diluted to a dilution of $10^{-10} \mathrm{~g} / \mathrm{mL}, 10^{-4}-10^{-10} \mathrm{~g} / \mathrm{mL}$ dilution of $B$. bassiana suspension, with a sterile pipette suck $100 \mu \mathrm{L}$ bacterial suspension, transferred to PDA medium, and evenly coated with a coating agent in the medium, $27{ }^{\circ} \mathrm{C}$ constant temperature culture $72 \mathrm{~h}$.

B. bassiana suspension preparation

According to the above culture results, it can be calculated that $1.5 \times 10^{11} \mathrm{cfu} / \mathrm{g}$ of $\mathrm{B}$. bassiana spores are contained in $1.0 \mathrm{~g}$ of $B$. bassiana spores and $0.02 \mathrm{~g}$ of $B$. bassiana is dissolved in $100 \mathrm{~mL}$ of PBS buffer to obtain a concentration. As $3 \times 10^{7} \mathrm{cfu} / \mathrm{mL}$ suspension of $B$. bassiana, recorded as $\mathrm{G}_{1}$; Pipette $10 \mathrm{~mL} \mathrm{G}_{1}$ suspension into $90 \mathrm{~mL}$ of PBS buffer solution to give a concentration of $3 \times 10^{6}$ cfu / $\mathrm{mL}$ suspension of $B$. bassiana, recorded as $\mathrm{G}_{2}$. The suspension of $B$. bassiana with the concentrations of $3 \times 10^{5} \mathrm{cfu} / \mathrm{mL}, 3 \times 10^{4} \mathrm{cfu} / \mathrm{mL}$ and $3 \times 10^{3} \mathrm{cfu} / \mathrm{mL}$ were obtained by the above methods and recorded as $\mathrm{G}_{3}, \mathrm{G}_{4}$ and $\mathrm{G}_{5}$ respectively.

\section{B. bassiana control pest detection}

The concentration of $\mathrm{G}_{1}-\mathrm{G}_{5}$ suspension of $B$. bassiana evenly applied to the Trialeurodes vaporariorum, Brevicoryne brassicae, Pieris rapae and Plutella xylostella larvae surface and PBS buffer solution instead of $B$. bassiana suspension as control group, 20 groups of experiments, 4 groups of control, observe the number of deaths and deaths and record analysis; all data were treated with SPSS 16.60 . 


\section{Result analyses}

\section{Effect of B. bassiana suspension on Trialeurodes vaporariorum}

According to the lethal test of $B$. bassiana suspension against $B$. tabaci larvae showed that different concentrations of $B$. bassiana suspension caused different mortality rates to B. tabaci larva, the increase of liquid concentration led to the increase of lethality of $T$. tabulaeformis larvae in conservatory. The lethal rates of $\mathrm{B}$. tabaci larvae in $\mathrm{G}_{1}$ and $\mathrm{G}_{2}$ were over $50 \%$ on the 5 th day, that is the lethality at half the lethal concentration and $\mathrm{G}_{3}$ concentration the lethality of $B$. tabaci larvae on the 5th day was $50 \%$ in suspension-infected larvae, while the lethality of $B$. tabaci larvae in $\mathrm{G}_{4}$ and $\mathrm{G}_{5}$ suspension did not reach $50 \%$, which was $30 \%$ and $38 \%$ respectively. Therefore, the $\mathrm{G}_{3}$ concentration B. bassiana suspension is the semi-lethal dose $\left(\mathrm{LD}_{50}\right)$ for this experiment (Figure 1).

\section{Effect of B. bassiana suspension on Brevicoryne brassicae}

According to $B$. bassiana suspension lethal test on Brevicoryne brassicae showed that: Different concentrations of $B$. bassiana suspensions of Brevicoryne brassicae lethal rate is different, $1-4 \mathrm{~d}$ period $\mathrm{G}_{1}-\mathrm{G}_{5}$ mortality curve is more gentle, from the 5th day, B. bassiana lethal to Brevicoryne brassicae significantly increased, of which $\mathrm{G}_{1}$ to Brevicoryne brassicae lethal rate as high as $63 \% \mathrm{G}_{3}$ on Brevicoryne brassicae lethality was $50 \%, \mathrm{G}_{5}$ Brevicoryne brassicae lethality was only $28 \%$. Therefore, the suspension of $B$. bassiana at $\mathrm{G} 3$ concentration is the $\mathrm{LD}_{50}$ for this experiment (Figure 2).

\section{Effect of B.bassiana suspension on Pieris rapae}

According to the B. bassiana suspension lethal to Pieris rapae results show that: B. bassiana suspension within 1-4 days had less effect on the mortality of Pieris rapae. On the 5th day, the mortality of B. bassiana to Pieris rapae was significantly higher than that of Pieris rapae in the first 4 days, and the mortality of $\mathrm{G}_{1}$ to Pieris rapae was $59 \%, \mathrm{G}_{2}$ bacterial suspension on cabbage worm mortality was $51 \%$, the lethality of $\mathrm{G}_{3}$ bacterial suspension to Pieris rapae was $48 \%$. Therefore, the suspension of $B$. bassiana at both $\mathrm{G}_{2}$ and $\mathrm{G}_{3}$ concentrations was the $\mathrm{LD}_{50}$ for this experiment (Figure 3).

Effect of B. bassiana suspension on Plutella xylostella

According to the B. bassiana suspension lethal to Plutella xylostella results show that: Mortality of Plutella xylostella larvae after different concentrations of $B$. bassiana suspension were different, and the lethal effect was obvious on the 5th day, among them, the lethal rate of $\mathrm{G}_{1}$ to Plutella xylostella larvae was as high as $62 \%$, the lethality of $\mathrm{G}_{2}$ suspension to Plutella xylostella larvae was $58 \%$, the lethality of $\mathrm{G}_{3}$ bacterium to Plutella xylostella larva was $51 \%$, The lethality of $\mathrm{G}_{4}$ suspension to Plutella xylostella larvae was $44 \%$, and the lethality of $\mathrm{G}_{5}$ suspension to Plutella xylostella larvae was only $34 \%$. Therefore, the concentration of $\mathrm{G}_{3}$ concanavalin suspension was the

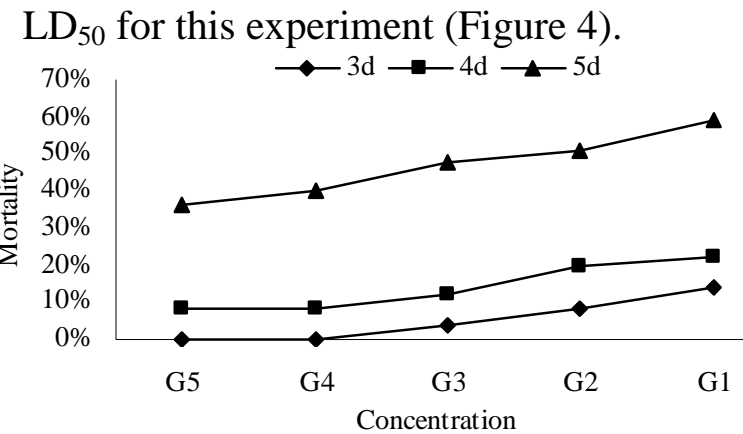

Figure 3. Mortality of cabbionella different concentrations of Beauveria bassiana to Pieris rapae

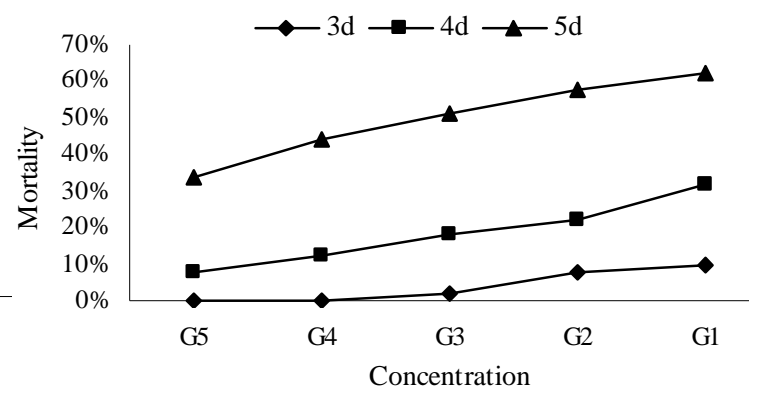

Figure 4. Lethality of Beauveria bassiana suspension to Plutella xy lostella larvae at different concentrations 


\section{Discussion}

B. bassiana suspension in conservatory Trialeurodes vaporariorum, Brevicoryne brassicae, Pieris rapae and Plutella xylostella larvae 4 kinds of cold conservatory common pests, have a significant lethal effect. The control group did not find lethal phenomenon. In the prevention and control experiment of conservatory beetle larvae, experimental mortality of $\mathrm{G}_{4}$ was lower than that of $\mathrm{G}_{5}$, because non-infected larvae existed in the suspension of B. bassiana at $\mathrm{G}_{4}$ concentration. The experimental data analysis shows that the concentration of $B$. bassiana suspension can be formulated into biopesticide between $G_{1}$ and $G_{5}$, but taking into account many factors such as control effect and dosage of biological pesticide, etc. They priority should be given to practical application $\mathrm{G}_{3}$. Since this experiment is done indoors, it has some limitations. Therefore, applying the results of this experiment to conservatory, we need to consider many influencing factors such as temperature and humidity, and conduct concrete multi-faceted studies on the basis of theory. Therefore, in the comprehensive management of conservatory pests, for this aspect of fungal control conservatory pests, this experiment can provide more detailed and accurate experimental basis and technical support.

\section{Acknowledgements}

Heilongjiang Provincial Nature Fund Project (C2017064); Heilongjiang Provincial Department of Education of the project (12531696);

\section{References:}

[1] Yao, Huaiying, Xiaodan Jiao, and Fengzhi Wu. "Effects of continuous cucumber cropping and alternative rotations under protected cultivation on soil microbial community diversity." Plant and Soil 284.1 (2006): 195-203.

[2] Krischik, Vera A., Alyson L. Landmark, and George E. Heimpel. "Soil-applied imidacloprid is translocated to nectar and kills nectar-feeding Anagyrus pseudococci Environmental Entomology 36.5 (2007): 1238-1245.

[3] Ranga Rao, G. V., et al. "Role of biopesticides in crop protection: present status and future prospects." Indian journal of plant protection 35.1 (2007): 1-9.

[4] Wang Li-min. Application Status, Existing Problems and Suggestions for Development of Biopesticides. Breeding technology consultant, 2011, (07): 261.

[5] Song Xi-gui, Liu Jian-yi. Plant Protection and Modern Science and Technology [J]. Middle school biology teaching, 2011, (09): 4- 6. 